my journal manuscript No.

(will be inserted by the editor)

\title{
Modelling transition phenomena of scientific coauthorship networks
}

\author{
Zheng Xie · Zhenzheng Ouyang · \\ Jianping Li · Enming Dong · Dongyun \\ Yi
}

Received: date / Accepted: date

\begin{abstract}
In a range of scientific coauthorship networks, transitions emerge in degree distribution, in the correlation between degree and local clustering coefficient, etc. The existence of those transitions could be regarded as a result of the diversity in collaboration behaviors of scientific fields. A growing geometric hypergraph built on a cluster of concentric circles is proposed to model two specific collaboration behaviors, namely the behaviors of research team leaders and those of the other team members. The model successfully predicts the transitions, as well as many common features of coauthorship networks. Particularly, it realizes a process of deriving the complex "scale-free" property from the simple "yes/no" decisions. Moreover, it provides a reasonable explanation for the emergence of transitions with the difference of collaboration behaviors between leaders and other members. The difference emerges in the evolution of research teams, which synthetically addresses several specific factors of generating collaborations, namely the communications between research teams, academic impacts and homophily of authors.
\end{abstract}

Keywords Coauthorship network · Hypergraph · Geometric graph · Modelling

\section{Introduction}

Scientific collaborations contribute not only to the breakthrough achievement unattainable by individual (Börner et al 2010, Jones, Wuchty \& Uzzi 2008), but also to the transmission and combination of knowledge (Adams 2012). In the scientometric perspective, coauthorship in scientific papers, as a valid proxy of the collaborations (Milojević 2010), can be expressed graphically by

Z. Xie, Z. Ouyang, J. Li, E Dong, D Yi

College of Science, National University of Defense Technology, Changsha, 410073, China

E-mail: xiezheng81@nudt.edu.cn 
the name of coauthorship networks, where nodes represent authors, and edges represent coauthor relationships. Since modern sciences increasingly involve collaborative research (Shrum, Genuth \& Chompalov 2007; Uzzi, Mukherjee, Stringer \& Jones 2013 Wuchty, Jones \& Uzzi 2007), the study of coauthorship networks has become an important topic of social science, especially of scientometrics (Glänzel \& Schubert 2004). It helps not only to understand the evolution and dynamics of scientific activities (Mali, Kronegger, Doreian \& Ferligoj 2014), but also to measure the contributions of scientists (Glänzel 2014), as well as to predict scientific success (Bertsimas, Brynjolfsson, Reichman \& Silberholz 2014; Sarigöl, Pfitzner, Scholtes, Garas \& Schweitzer 2014), etc.

The empirically observed coauthorship networks have specific common local (degree assortativity, high clustering) and global (power-law degree distribution, short average distance) properties (Newman 2001a b c, 2004), according to which they are marked as scale-free and small-world networks. Some important models have been proposed to reproduce those properties, such as modeling the scale-free property through preferential attachment (Barabási, Jeong, Néda, Ravasz, Schubert \& Vicsek 2002; Börner, Maru \& Goldstone 2004; Moody 2004; Perc 2010; Tomassini \& Luthi||2007; Wagner \& Leydesdorff 2005) or cumulative advantage (Milojević 2014), modeling the degree assortativity by connecting two non-connected nodes that have similar degrees (Catanzaro, Caldarelli \& Pietronero 2004).

One explanation for the power-law tails of degree distributions geos to the inhomogeneous influences of nodes: nodes with wider influences are likely to gain more connections. A specific example is that authors with large academic impacts, which often occupy a small fraction of the total authors in empirical coauthorship networks, capture voluminous collaborators. When using geometric graph theory (RGG) (Krioukov, Kitsak, Sinkovits, Rideout, Meyer \& Boguna 2012; Penrose 2003) to analyze networks, such as citation networks, web-graphs, the impacts of nodes in scientific research or Internet can be modeled by attaching specific geometric zones to nodes (Xie, Ouyang, Liu \& Li 2016; Xie, Ouyang, Zhang, Yi \& Kong 2015, Xie \& Rogers 2016). The same case works with the geometric graph model for coauthorship networks (Xie, Ouyang \& Li 2016), which is built on a circle and reproduces the aforementioned features of coauthorship networks at certain levels.

Besides the academic impacts, the homophily of authors in the sense of geographical distances and research interests turns out to be another factor of generating collaborations (Hoekmana, Frenken \& Tijssen 2010, Newman 2002). Compared with topological graph models, our previous model demonstrates an advantage of it expressing the homophily by spatial coordinates of nodes (Xie, Ouyang \& Li 2016). However, this model generates all authors at one time, and consequently fails to express the formation process of coauthorship networks. A growing geometric hypergraph is proposed here to model this process. It is built on a cluster of concentric circles, where each circle has a time coordinate. 
The proposed model imitates the collaborations in and between research teams in a dynamic way. The main collaborations occur in the same research team, the mechanism of which synthetically expresses the influences of the homophily and the academic impacts of authors on collaborations in geometrical ways. Our analysis demonstrates that the model can also capture the aforementioned features of the empirical data.

Interesting phenomena of the empirical data are the transitions emerged in degree distributions $P(k)$, average local clustering coefficient and average degree of neighbors as functions of degree $(C(k), N(k))$. The data features are different in the two regions of $k$ splitted by cross-over regions or tipping points. For example, the $P(k)$ of each empirical dataset emerges a generalized Poisson and a power-law in small and large $k$ regions respectively, where there exists a cross-over between the two regions. Our model successfully reproduces the shapes of those functions as well as their transitions, and gives reasonable explanations for those transitions.

To follow up the above, components of authors with voluminous collaborators are analyzed. Members of large "paper teams" (each team consists of a group of authors in a paper) are authors with large degree. It is found that when removing large paper teams, the degree distributions still have a power-law tail. Our model provides a reasonable explanation for the finding: the power-law tails are caused by the papers with many authors as well as the leaders of large research teams.

This report is organized as follows: the model and data are described in Sections 2 and 3 respectively; the degree distribution, clustering and assortativity are analyzed in Sections 4 and 5 respectively; the conclusion is drawn in Section 6.

\section{The model}

The model processes

In reality, most researchers belong to research teams in universities and research institutes. For each research team, one or several researchers are responsible for the running of the team as leaders. Research teams and their leaders are the main objects in our model, which have been used in Reference (Xie, Ouyang \& Li 2016). The term "article team" in Reference (Milojević 2014) is also adopted by our model, which is renamed as "paper team".

Our model creates "authors" (nodes) through a unit intensity Poisson process on a cluster of concentric circles. The circle cluster could be viewed as a "topic" or "interest" space. Note that it is not a real topic or interest space, which is a high dimensional space representing textual contents of authors' papers. In the model, some nodes are randomly selected as "leaders" (called lead nodes) to attach specific geometric zones imitating their academic impacts. For each lead node, its "research team" is formed by the nodes within its influential zone (Fig. 1). Unlike the "lead authors" who are in charge of 


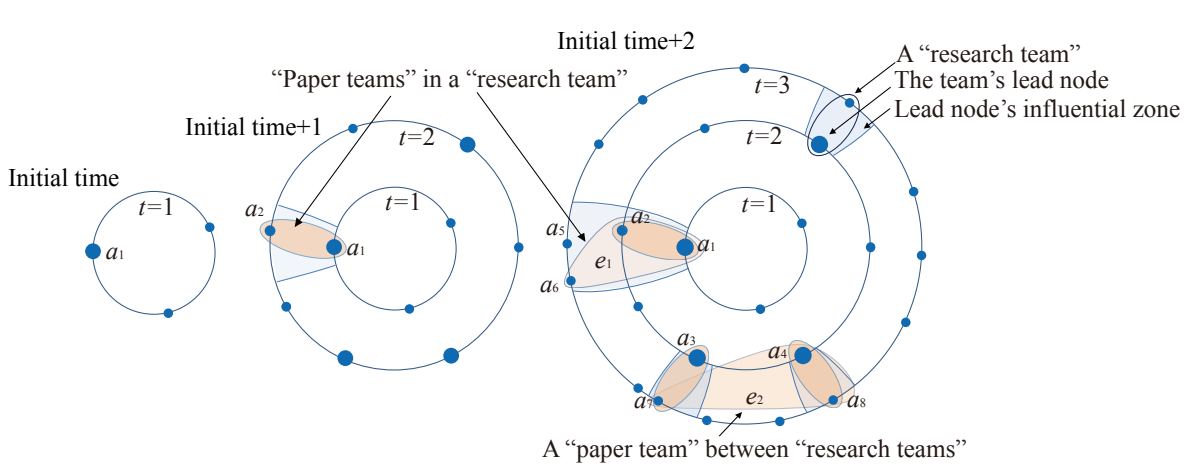

Fig. 1. Illustration of the model. The lead nodes (large nodes) have zones representing their academic impacts, the sizes of which change over time. The set of nodes in a zone (blue area) is regarded as a "research team", and the set of nodes in a hyperedge (orange area) as a "paper team". The sizes of "research teams" are in proportion to the corresponding geometric sizes.

"article teams" in S. Milojević's model (Milojević 2014), the lead nodes in our model are in charge of "research teams", and concurrently play the roles of "lead authors" in "article teams".

Inspired by the processes of generating coauthorship networks, the model generates hypergraphs first (in which nodes are regarded as "authors" and hyperedges as "paper teams"), then extracts simple graphs from the hypergraphs (in which edges are formed between every two nodes in each hyperedge). Note that the isolated nodes are ignored, and the multiple edges are viewed as one.

Since new papers are published per week or month, coauthorship networks evolve over time. Our model aims at simulating the evolution processes, especially the self-organizing formation of research teams in the processes. For this purpose, the numbers of hyperedges and nodes in the model are growing over time $t$. Parameter $t$ can be explained as the $t$-th unit of time, such as $t$-th week, $t$-th month, etc.

Empirical distributions of paper team sizes have a hook head and a fat tail (Fig. 22). Treating a paper team as a "space" for collaboration, a researcher joins a paper team can be treated as an event occurring in the space. The frequency of events occurring in a space follows the Poisson distribution, if these events independently occur at a fix rate. However, events of joining a paper team would be dependent. Paper team Sizes also vary over disciplines, for instance, they are large in biology, and small in mathematics. Those make the sizes of most paper teams follow the generalized Poisson distribution (which allows the occurrence probability of an event to be affected by previous events (Consul \& Jain 1973)), so form the hooks. There also exists a small fraction of very large paper teams, which appears as fat tails in the paper team size distributions. Those tails can be sufficiently fitted by power-law distributions. 

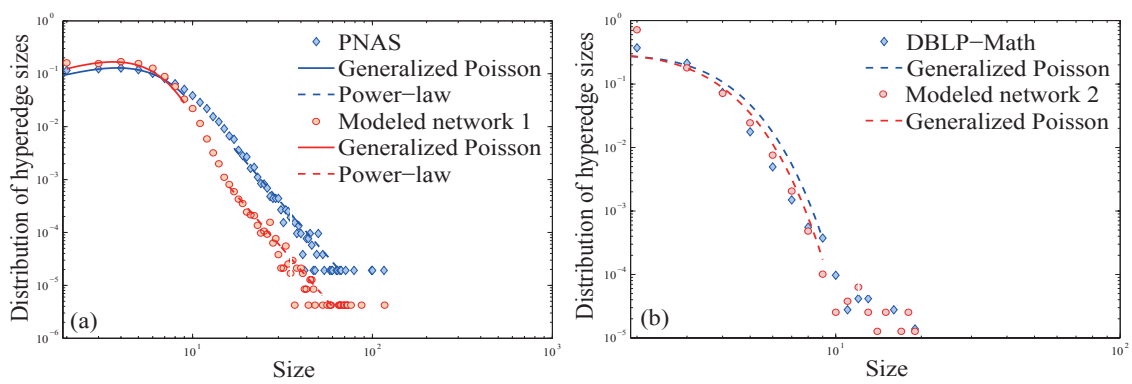

Fig. 2. The distributions of hyperedge sizes. Panels show the distributions of two modeled hypergraphs (parameters of which are listed in Section 3), PNAS 1999-2013 and DBLP-Math 1956-2013 respectively. The root mean squared errors (RMSE, used for measuring the goodness of fit) are 0.015 (generalized Poisson), 0.002 (power-law) for PNAS. 0.015 (generalized Poisson), 0.002 (power-law) for Modeled network 1, 0.113 for DBLP-Math, and 0.113 for Modeled network 2 .

Denote the probability density function (PDF) of paper team sizes by $f(x)$, $x \in \mathbb{Z}^{+}$. The PDFs of generalized Poisson and power-law are formulized as $f_{1}(x)=a(a+b x)^{x-1} \mathrm{e}^{-a-b x} / x !$ and $f_{2}(x)=c x^{-d}$ respectively, where $a, b, c, d \in$ $\mathbb{R}^{+}$, and $x$ belongs to a subset of $\mathbb{Z}^{+}$. We can generate random variables of an $f(x)$ with head $f_{1}(x)$ and tail $f_{2}(x)$ by sampling random variables of $f_{1}(x)$ and $f_{2}(x)$ with probability $q$ and $1-q$ respectively. The modeled hyperedge sizes are generated from a given $f(x)$.

Two oversimple assumptions made here to simplify the modelling process. (1) The linear growth of "authors" could not hold in reality. If changing it, the formula of influential zones should be changed to capture features of empirical data. (2) In the second connection rule, grouping together certain numbers of nodes with the same degree is the simplest expression of the degree assortativity of authors in empirical data: authors prefer to collaborate with other authors with similar degrees (Newman 2002). More reasonable mechanism of degree assortativity still needs further research.

Based on above preparations and assumptions, we build a hypergraph on a cluster of concentric circles $S_{t}^{1}, t=1,2, \ldots, T\left(T \in \mathbb{Z}^{+}\right)$as follows:

1. Coordinate and influential zone (simply "zone hereafter) assignment

For time $t=1,2, \ldots, T$ do:

Sprinkle $N_{1}$ nodes as "potential authors" uniformly and randomly on a circle $S_{t}^{1}$. Identify each node, e. g. $i$, by its spatio-temporal coordinates $\left(\theta_{i}, t_{i}\right)$, where $t_{i}$ is the generating time of $i$;

Select $N_{2}$ nodes from the new nodes randomly as lead nodes to attach specific zones: the zone of a lead node, e. g. $j$, is defined as an interval of angular coordinate with center $\theta_{j}$ and length $\alpha\left(\theta_{j}\right) t_{j}^{-\beta} t^{\beta-1}$, where $\alpha\left(\theta_{j}\right)$ is a piecewise constant non-negative function of $\theta_{j} \in[0,2 \pi)$, and $\beta \in[0.5,1]$;

2. Connection rules (simply "Rule" hereafter) 
For time $t=1,2, \ldots, T$ do:

(a) For each new node $i$, search the existing lead nodes whose zones cover $i$. For each such lead node $j$, generate a hyperedge with size $m$ by grouping together $i, j$ and $m-2$ neighbors of $j$ nearest to $i$, where $m$ is a random variable drawn from a given $f(x)$ or the number of $j$ 's neighbors plus two if the former is larger than the latter.

(b) Select $N_{3}$ existing nodes (no distinction is made between lead nodes and the others) with non-zero degree randomly. For each selected node $l$, generate a hyperedge by grouping together $l$ and $m-1$ randomly selected nodes with the same degree of $l$, where $m$ is a random variable drawn from a given $f(x)$ or the number of nodes with the same degree of $l$ if the former is larger than the latter.

Here randomly selecting means sampling without replacement. In reality, most paper teams are subsets of research teams, which are often formed by a leader and some team members who have similar research interests. This collaboration mode is imitated by Rule (a), which groups together a certain number of nearest nodes and their lead node as a hyperedge. Meanwhile, a few paper teams are unions of some subsets from different research teams, even from different countries (Glänzel 2011). Such teams are very likely to appear in interdisciplinary papers, which account a relatively small fraction of total papers. For example, the proportion of the papers marked as interdisciplinary ones in PNAS 1999-2013 is 5.7\% (Xie, Duan, Ouyang \& Zhang 2015). The collaborations between research teams are modeled by Rule (b), which gives a possibility to connect the nodes in different research teams. Researchers can join different research teams. This phenomenon also is equivalently imitated by Rule (b) to some extents.

\section{Innovation of the model}

The improvements in the ability to reproduce empirical features (which will be shown in following sections) are achieved by the new features of the model, rather than by better selection parameters of our previous model (Xie, Ouyang \& $\mathrm{Li}$ 2016). For example, the way of generating hyperedges is different. A new node will "coauthor" with its lead nodes, and some existing nodes in the lead nodes' zones, which are nearest to it in the sense of space. Therefore, in the end, an older node (which could be non-lead node) can generate hyperedges together with the nodes which are not its nearest neighbors, since the nearest neighbors may not be generated when the hyperedges were generated. This difference causes the diversity of ages not only for lead nodes (which is addressed by the previous model), but also for other nodes. As shown in Fig. 1, when the new node $a_{6}$ at time $t=3$ generates a hyperedge (which contains three nodes), it should "coauthor" with its lead node $a_{1}$ and one nearest existing nodes in the zone of $a_{1}$, namely $a_{2}$, but not the nearest one $a_{5}$.

Note that there exists a difference between Barabási-Albert (BA) model (Barabási, Jeong, Néda, Ravasz, Schubert \& Vicsek 2002) and our model. In BA model, 
nodes make decisions to connect old nodes with a probability that is proportional to the degrees of nodes. Hence, those decisions are made based on knowing the degree information of all nodes, and so they are global behaviors. In our model, most of the connection decisions made by nodes are restricted by geometric locations, and so are local behaviors. Those decisions are imitated by Rule (a). A few decisions are made randomly, which are modelled by Rule (b). In reality, authors making decisions to choose collaborators has the locality of geography and research interests, as well as certain uncertainty or randomness.

The model expresses the sizes of real leaders' academic impacts and those of their research teams by the sizes of lead nodes' influential zones. For a lead node $i$, the size of its "research team" (the number of members in its zone) is $\delta \alpha\left(\theta_{i}\right) t_{i}^{-\beta} t^{\beta-1}$ at time $t>t_{i}$, where $\delta=N_{1} /(2 \pi)$. Hence the cumulative number of nodes in the zone is

$$
n\left(\theta_{i}, t_{i}, t\right)=\delta \times\left(\sum_{s=t_{i}+1}^{t} \alpha\left(\theta_{i}\right) t_{i}^{-\beta} s^{\beta-1}\right) \approx \frac{\alpha\left(\theta_{i}\right) \delta}{\beta}\left(\frac{t}{t_{i}}\right)^{\beta} .
$$

There are some intuitive explanations for Formula 1. Firstly, the value of $t-t_{i}+1$ is interpreted as the scientific age of a researcher, namely a researcher spending more time on research would have a larger academic impact and more collaborators as well. Hence it is reasonable to consider the sizes and cumulative sizes of research teams as increasing functions of scientific age, consequently decreasing functions of $t_{i}$. Secondly, the cumulative sizes of research teams will increase over $t$ due to the continuously coming collaborators (e. g. tutors may have new students every year). Thirdly, the research team sizes may be different in research fields, so $\alpha(\cdot)$ is introduced to the formula. In addition, academic impacts of a leader, so the increment of cumulative research-team size could be considered to shrink over time $\left(\partial^{2} n\left(\theta_{i}, t_{i}, t\right) / \partial t^{2}<0\right)$ due to the process of retirements or activity decreases. Unlike papers, the aging of authors appears only for the data with large time span. Hence, the major role of the factor $t^{\beta-1}$ in the formula of zonal sizes is to tune the exponent of power-law.

A weakness of our model is that it has a lot of parameters. The $f(k)$ in connection rules tunes the distribution of paper-team sizes. Parameters $\alpha(\cdot)$ and $\beta$ tunes the distribution of collaborators per author. There is no parameter directly tunes the average local clustering coefficient and average neighbor degree. Hence the remarkable model-data fits of those properties are impressive to us.

\section{The data}

The empirical data

In order to test the universal reproduction ability of the proposed model, we analyze two empirical coauthorship networks from two metadata with different 
collaboration levels (Table 2). One is DBLP-Math, which is constructed from 72,269 papers published in 54 mathematical journals during 1956-2013. The data are obtained from DBLP database (http://dblp.uni-trier.de/xml/dblp). The other one is PNAS, which is constructed from 52,803 papers published in the Proceedings of the National Academy of Sciences (http://www.pnas.org) during 1999-2013.

In the process of extracting networks from those metadata, authors are identified by their names on their papers. For example, the author named "Carlo M. Croce" on his paper is represented by the name. We mainly focus on the degree distribution of network and some properties based on degree. The coauthorship network from the papers in PNAS 2012 is analyzed in Reference (Kim \& Diesner 2016). From the analysis, we find that identifying authors by their name on papers does not change the ground truth distribution type, which partially verifies the reliability of the empirical networks used here.

In order to analyze the components of authors with large degrees, SubPNAS, a sub-network of PNAS, is extracted from the papers, the numbers of authors in which are less than the boundary point of the generalized Poisson part in the distribution of hyperedge sizes. An algorithm is provided to detect boundary points of PDFs by using specific statistical technologies synthetically (Table 11). Inputs are paper-team sizes, $g(\cdot)=\log (\cdot)$ and $h(\cdot)=f_{1}(\cdot)$. Using $\log (\cdot)$ can rescale the differences between the fitting model and empirical data at different scales, which helps to detect the boundary points at small scales.

Table 1. Boundary point detection algorithm for PDFs.

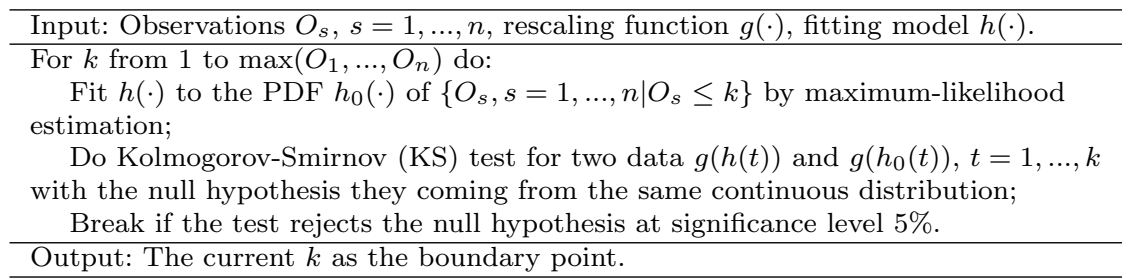

The synthetic data

Two synthetic coauthorship networks are generated to reproduce several properties of the empirical data. For Modeled network $1(2), q=0.9625(0.999)$ in Rule (a), $q=1$ (0.999) in Rule (b), $f_{1}(x)$ is the Poisson distribution with mean $5.5(2.3)$, and $f_{2}(x) \propto x^{-3.7}, x \in[10,150]([11,20])$ in Rules (a-b). Set $T=4,500(9,000)$ and $N_{1}=100(15)$ to make the number of nodes comparable to that of the empirical data in magnitude. Set $N_{2}=N_{1} / 5, \alpha=0.19$ $(0.2)$ and $\beta=0.42(0.43)$ to make the average degree comparable to that of the empirical data. Set $N_{3}=1(0.5)$ to make the generated network have a 
giant component and the node proportion of the giant component comparable to that of the empirical data.

There are some reasons for choosing such parameters. The degrees of nodes in the empirical data are not very large. So the value of $\alpha(\cdot)$ should be small, when $N_{1}$ is large. In reality, the leaders occupy a small fraction of the total researchers (potential authors), so $N_{2}$ is set to be far less than $N_{1}$. Meanwhile, the number of paper teams within a research team is far more than that between research teams, so $N_{3}$ is set to be small. In practice, $N_{3}$ could be a decimal belonging to the interval $[0,1]$, which means implementing Rule (b) under probability $N_{3}$ at each time step. Since the model is stochastic, we generate 20 networks with the same parameters, and compare their statistical indicators in Table 2. The finding is that the model is robust on those indicators.

Table 2. Specific statistical indicators of the analyzed networks.

\begin{tabular}{lrrrrrrrr}
\hline Network & NN & NE & GCC & AC & AP & MO & PG & NG \\
\hline PNAS & 201,748 & $1,225,176$ & 0.881 & 0.230 & 5.736 & 0.884 & 0.868 & 4,848 \\
DBLP-Math & 68,183 & 99,116 & 0.756 & 0.157 & 9.256 & 0.935 & 0.477 & 15,492 \\
Modeled network 1 & 193,655 & $1,261,131$ & 0.788 & 0.228 & 5.957 & 0.952 & 0.817 & 6,230 \\
Modeled network 2 & 70,921 & 121,685 & 0.687 & 0.095 & 9.429 & 0.946 & 0.606 & 8,940 \\
Sub-PNAS & 200,170 & $1,158,503$ & 0.881 & 0.097 & 5.806 & 0.882 & 0.867 & 4,869 \\
\hline
\end{tabular}

The indicators are the numbers of nodes (NN) and edges (NE), global clustering coefficient (GCC), assortativity coefficient (AC), average shortest path length (AP), modularity (MO, calculated by the Louvain method (Blondel, Guillaume, Lambiotte \& Lefebvre 2008), the node proportion of the giant component (PG), and the number of components (NG). The values of AP of the first, third and fifth networks are calculated by sampling 15,000 pairs of nodes.

Specific features in common

The indicator modularity in Table 2 shows that the same as the empirical networks, the modeled networks have clear communities. The reason is that nodes in the same "research team" probably belong to the same community due to Rule (a), and the fraction of connections between "research teams" is small due to Rule (b). Thus edges within communities are significantly more than those between communities, which results to the clear community structures.

In reality, a leader of a research team often collaborates with all of the team members. Hence the leader acts as a hub in the sub-network of coauthorship restricted in the leader's research team. The communications between research teams make empirical coauthorship networks have a giant component. Hence, authors evolving in the communications (e. g. visiting scholars or students) also act as hubs in macroscopic scale. Therefore, it is reasonable to regard that bi-level (maybe multilevel) hub structures exist in coauthorship networks. 

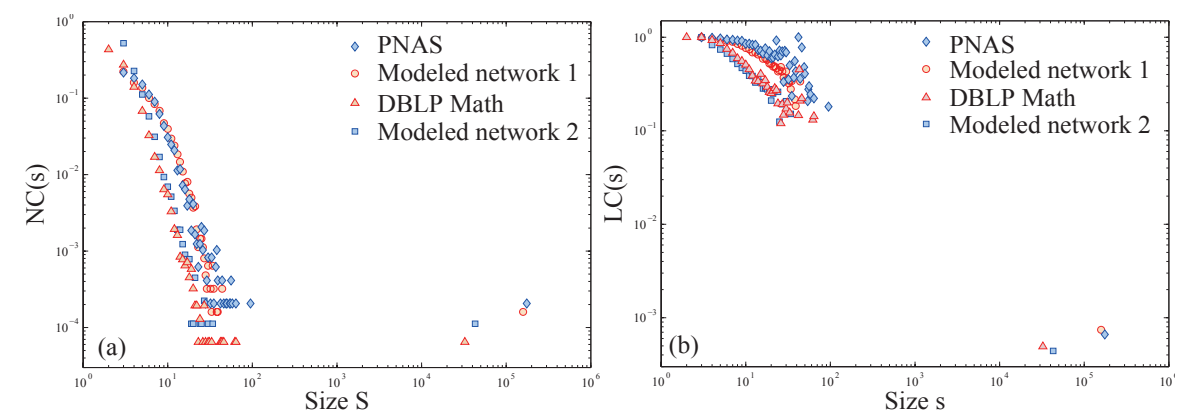

Fig. 3. The distributions of component sizes $N C(s)$ and the average proportion of the largest cliques in $s$-components (components with size $s) L C(s)$ of the first four networks in Table 2, If there is an $s$ that makes $L C(s) \approx 1$, it means the $s$-components are nearly fully connected.

In the model, due to Rule (a), a lead node also plays the role of hub in the subgraph restricted in its zone, because all of the nodes in the zone connect to the lead node. Due to the randomly selection in Rule (b), the nodes in large zones are preferably connected by the nodes of other zones, which makes the nodes in the large zones hubs for the nodes in the small zones. Hence the modeled networks also have a bi-level hub structure, which is a reason for that the average shortest path length of each empirical or synthetic network scales as the logarithm of the number of nodes (Table 2).

Coauthorship networks, in essential, are hypergraphs, which leads the high clustering coefficient. An author of a paper connecting to the other coauthors generates very many triangles. Together with the average shortest path length scaling as the logarithm of the number of nodes, the synthetic networks can be regarded having the small-world property of the empirical networks.

In the model, a subgraph restricted in a zone will form a component by itself, if its nodes are not selected by Rule (b). The distributions of component sizes are similar to those of the empirical data (Fig. 3 a), which validates the reasonability of taking the zonal sizes from a power-law function.

Authors in small research teams (no larger than the mean size of paper teams) are more likely to write papers together. With the growth of researchteam-sizes, some authors will stop writing, which causes the average proportion of the largest cliques in a component decreases with the growth of component size (Fig. 3b). The model also captures this feature, which reinforces the reasonability of model design.

First, the number of edges did not change much. On average, FD and HD reduced the number by $1.20 \%$ and $0.17 \%$, respectively, while $\mathrm{AD}$ increased it by $0.11 \%$. Overall, the number of edges can be seen to be constant. This suggests that merged author nodes typically have distinct sets of coauthors. If two merged author identities have coauthors that are also merged due to name ambiguity, then the ties between each author and coauthor would also be merged, leading to the decrease of ties. 
Second, density showed a tendency to increase by initial-based disambiguation. Network densities of PGT networks increased by on average $47.52 \%$ (FD), $14.93 \%$ (AD), and $20.72 \%$ (HD). Density has a characteristic to decrease with the network size. The decrease of numbers of unique authors by initialbased disambiguation seems to mostly affect the increase of densities because numbers of edges are almost constant as shown above.

The average degree increased in all datasets (avg. $20.23 \%$ by FD, $7.12 \%$ by $\mathrm{AD}$, and $9.63 \%$ by $\mathrm{HD}$ ) with increased standard deviations. We can infer that merged authors were connected to others who are not their actual collaborators, contributing to the inflation of average coauthor size.

The size of largest components also increased by on average $37.26 \%$ by FD, $24.08 \%$ by $\mathrm{AD}$, and $30.11 \%$ by $\mathrm{HD}$, while the number of components decreased in all cases. As authors were merged into other identities, they attached their local networks to others, thus increasing the component size. Some fields showed noticeable increases which might lead to different interpretation of the coauthorship network structure. For example, in biology, the largest component in FD data contained $49.25 \%$ of all authors, while the largest component of its PGT data contained $0.72 \%$. PNAS also showed the same observation. From the perspective of FD, the collaboration network in biology and PNAS dataset is way more inter-connected and mature than it actually is.

\section{Modeling the transition of degree distributions}

\section{Features of degree distributions}

The degree distributions of coauthorship networks (the distributions of collaborators per author) appear two common features, namely a hook head and a fat tail, which can be sufficiently fitted by generalized Poisson and power-law distributions respectively (Fig. 4). The boundary points of generalized Poisson parts in degree distributions are detected by the algorithm in Table 1. Inputs are degrees as observations, $g(\cdot)=\log (\cdot)$ and $h(\cdot)=f_{1}(\cdot)$.

In statistics, if regarding authors of a coauthorship network as samples, such a mixture distribution means those samples come from different populations, namely the collaboration mode of authors with small degrees differs from that with large degrees. In reality, authors mainly are teachers and students in institutes and universities, who can be viewed as two distinct populations. The collaboration modes of students and teachers are different. Many students only write a few papers, and do not write after graduations, but their teachers could continuously write papers collaborating with their new students or other researchers.

There are two essential questions for the emergence of such degree distributions: why the distributions emerge generalized Poisson and power-law; is there any essential relation between them? We attempt to give an answer by analyzing and simulating collaboration modes as follows. 


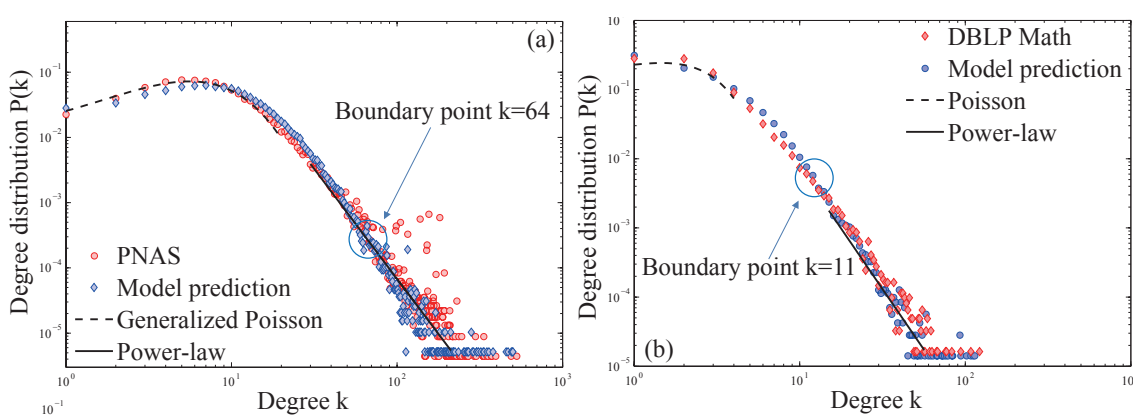

Fig. 4. The degree distributions of the first four networks in

Table 2. The fitting functions are the PDFs of generalized Poisson and power-law for the heads and tails respectively. The RMSEs are 0.01 (generalized Poisson), 0.019 (power-law) for PNAS and 0.009 (Poisson), 0.02 (power-law) for DBLP-Math. The fittings pass the KS test at significance level $5 \%$.

Collaboration behaviors are dependent on authors' choices. We simply treat the choices (e. g. whether or not a researcher joins a research team) as "yes/no" decisions. So the size of a research team is equal to the number of successes in a sequence of $n$ decisions, where $n$ is the number of candidates for the members of the research team. Approximate the probability $p$ of "yes" by its expected value $\hat{p}$, and suppose those "yes/no" decisions to be independent. Then, the sizes of research teams will follow a binomial distribution $B(n, \hat{p})$. When $n$ is large and $\hat{p}$ is small, $B(n, \hat{p})$ can be approximated by a Poisson distribution with mean $n \hat{p}$ (Poisson limit theorem). The value of $n \hat{p}$ is not a constant due to the diversity of research teams' attractive abilities.

In reality, the "yes/no" decisions could be affected by previous occurrences, e. g. students sometimes introduce their research teams to their juniors. So for small research teams, it is reasonable to regard their sizes as random variables drawn from a generalized Poisson distribution (which allow the probability of an event's occurrence to affect by previous events (Consul \& Jain 1973)).

For large research teams, the numbers of their candidates are large enough that the "yes/no" decisions can be regarded to be independent. So their sizes could be regarded as random variables drawn from a range of Poisson distributions with sufficiently large means. The diversity of attractive abilities of research teams gives the possibility of a few research teams having highly attractive abilities, and then guarantees the relative commonness for a few authors getting collaborators that greatly exceed the average. The commonness reflects as a feature of the distribution with a power-law tail, or asymptotically. 
Modelling the features

The analytical derivations (in Appendix) and numerical evidences (the blue diamonds in Fig. 4) illustrate the ability of our model in reproducing the empirical degree distributions. The tunable model parameters give our model flexibility for empirical networks in diverse fields. Specifically, the power exponents of fat tails and the hook peaks can be tuned by $\beta$ and the expected value of $f_{1}$ respectively. In what follows, an intuitionistic explanation is given to show how the geometric aspect of the model is actually necessary to fit the empirical degree distributions.

In our model, nodes are generated according to a Poisson point process, hence the number of nodes covered by a zone is a random variable drawn from a Poisson distribution with an expected value in proportion to the zonal size. Our model generates a range of Poisson distributions with means taking values from a power-law function.

Now we analyze how the emergence of generalized Poisson is captured by our model. The nodes with small degrees usually belong to only one small hyperedge, or come from small "research team", the sizes of which are no larger than the mean size of hyperedges. For the first case, the empirical data show that the heads of hyperedge size distributions are well fitted by generalized Poisson (Fig. 2). Hence the degrees of nodes which belong to only one small hyperedge are equal to the size of the hyperedge minus one, hence also follow a generalized Poisson distribution. For the second case, nodes in those small "research teams" probably belong to one hyperedge. Ignoring the minority of connections between "research teams" generated by Rule (b), the degrees of those nodes are the sizes of corresponding "research teams" minus one, therefore also follow Poisson distributions. Therefore the heads of the modeled degree distributions follow a mixture distribution, and can be well fitted by a generalized Poisson distribution with proper parameters.

Next we turn to the power-law. The nodes with large degrees are the nodes of large hyperedges or the lead nodes of large research teams. Consider the first case. The tails of the input hyperedge-size distributions follow a power-law distribution, as the empirical data do. For example, in PNAS, the sizes of large hyperedges follow a power-law distribution with exponent $\gamma=-3.96$ (Fig. 2). If supposing each node belongs to only one of such hyperedges, then the degrees of those nodes are drawn from a power-law distribution with exponent $\gamma-1$. However, the supposition is not fully established in reality (Fig. 5b). Consider the second case. The lead nodes usually "collaborate" with all of their team members. Ignoring the minority of "collaborations" between "research teams", the degree of a lead node is the size of its "research team" minus one. A power-law can appear when averaging a range of Poisson distributions with expected values from a power-law function. Our model generates such Poisson distributions by making the sizes of influential zones from a power-law function, which gives a sufficient diversity for lead nodes' attractive abilities. In fact, the scale-free property of the modeled networks is hidden in the diverse sizes, which is the reason for the remarkable data-model fit (Fig. 4). 

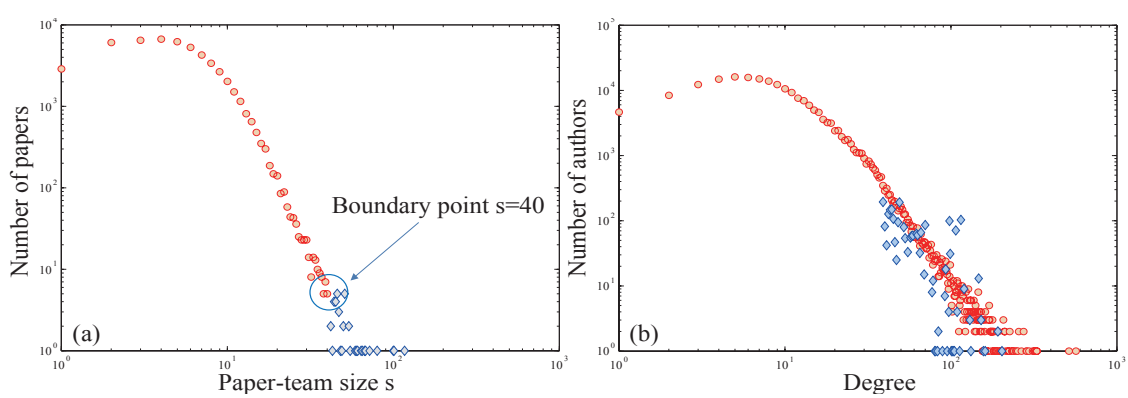

Fig. 5. The components of the authors with many collaborators. Panel (a) shows the distribution of paper-team (hyperedge) sizes and its boundary point (the detecting way of which is described in Section 3) for PNAS 1999-2013. The red circles and blue diamonds in Panel (b) express the degree distributions of the networks extracted from the paper teams with sizes $s<40$ and $s \geq 40$ respectively.

The mathematical deduction of generating power-law from Poisson is proposed in Appendix, where the calculations in Eq. 4 are inspired by some of the same general ideas as explored in the cosmological networks (Krioukov, Kitsak, Sinkovits, Rideout, Meyer \& Boguna 2012). In fact, the deduction illustrates the relation between the Poisson and power-law. It shows that "scale-free", namely the emergence of power-law tails, partly comes from many Poisson processes, consequently from many "yes/no" decisions. In this sense, coauthorship networks give good examples of " $1+1>2$ " for systems science and complexity.

The transition from Poisson to power-law is smooth in DBLP-Math, but not in PNAS (Fig. 4). A reason for the difference is that the components of large degree nodes in the two datasets are different. Authors with large degrees in PNAS partly come from large paper teams (Fig. 5), but DBLPMath has no large paper team. If an author only writes papers with small paper teams, the growing process of his/her degree is smooth. In our model, the growing process is represented by the smoothly increasing process of each research team's cumulative size (Eq. 1). Meanwhile, model parameter $q$ tunes the proportion of large paper teams. Hence, when $q$ is small, the transitions of modeled networks, e. g. Modeled network 2, are smooth.

\section{Modelling the transitions in clustering behavior and degree assor- tativity}

Transitions in clustering behavior and degree assortativity

As global features, the positive Pearson correlation coefficient of degrees between pairs of collaborated authors (degree assortativity) and high global clustering coefficient (the fraction of connected triples of nodes which also form 
"triangles") are common in coauthorship networks. A general interpretation for degree assortativity of social networks is the homophily of nodes, namely similar people attract one another (Newman 2002). The homophily in research interests is the precondition of collaborations. The homophily is also an explanation for high global clustering coefficient, because the relation of similarity between nodes is symmetric, reflexive and transitive.

It can be found that the clustering behavior and degree correlation differ from the authors with small degrees to those with large degrees. Denote the average local clustering coefficient and average neighbor degree of $k$-degree nodes by $C(k)$ and $N(k)$ respectively. Degree correlation can be measured by the slope of $N(k)$ (Pastor-Satorras, Vázquez \& Vespignani 2001). If the function is increasing, nodes with large degrees connect to nodes with large degrees on average, which means the network is assortative.

There exist transitions in the $C(k)$ and $N(k)$ of the empirical data (Fig. 6 Fig 7). The tipping points of those functions are detected by the algorithm in Table 3. The inputs are $C(k) / N(k), g(\cdot)=\log (\cdot)$ and $h(s)=a_{1} \mathrm{e}^{-\left(\left(s-a_{2}\right) / a_{3}\right)^{2}} /$ $h(s)=a_{1} s^{3}+a_{2} s^{2}+a_{3} s+a_{4}\left(s, a_{i} \in \mathbb{R}, i=1, \ldots, 4\right)$. Using those inputs is based on the observation of $C(k)$ and $N(k)$. Using the term "tipping point" is suitable for PNAS data, because the features in the two regions splitted by the points have significant difference. However, the term is not quite accurate for the $C(k)$ of DBLP-Math, because its transition is not so sharp. Using boundary point may be more suitable.

When $k$ is larger than the tipping point, $C(k)$ of each empirical network emerges a decreasing trend, which is proportional to $1 / k$. Meanwhile, the correlation coefficients of $k$ and $N(k)$ in the two regions of $k$ splitted by tipping points are $0.416 /-0.025$ and $0.250 /-0.046$ for PNAS and DBLP-Math respectively. The existence of tipping points in $C(k)$ and $N(k)$ provides an evidence for the difference between the collaboration behavior of authors with small degrees and that with large degrees.

Table 3. Boundary point detection algorithm for general functions.

\begin{tabular}{l}
\hline Input: Data vector $h_{0}(s), s=1, \ldots, K$, rescaling funtion $g(\cdot)$, fitting model $h(\cdot)$ \\
\hline For $k$ from 1 to $K$ do: \\
Fit $h(\cdot)$ to $h_{0}(s), s=1, \ldots, k$ by regression; \\
Do KS test for two data vectors $g(h(s))$ and $g\left(h_{0}(s)\right), s=1, \ldots, k$ with the null \\
hypothesis they coming from the same continuous distribution; \\
Break if the test rejects the null hypothesis at significance level $5 \%$. \\
Output: The current $k$ as the boundary point.
\end{tabular}

The low local clustering coefficients of large degree nodes cannot be explained by homophily, so does and non-positive correlation coefficients of degree $k$ and $N(k)$ in large $k$ regions. An explanation is given as follows. The analysis in above section has illustrated that authors in small research teams (no larger than the mean size of paper teams) are more likely to write papers 

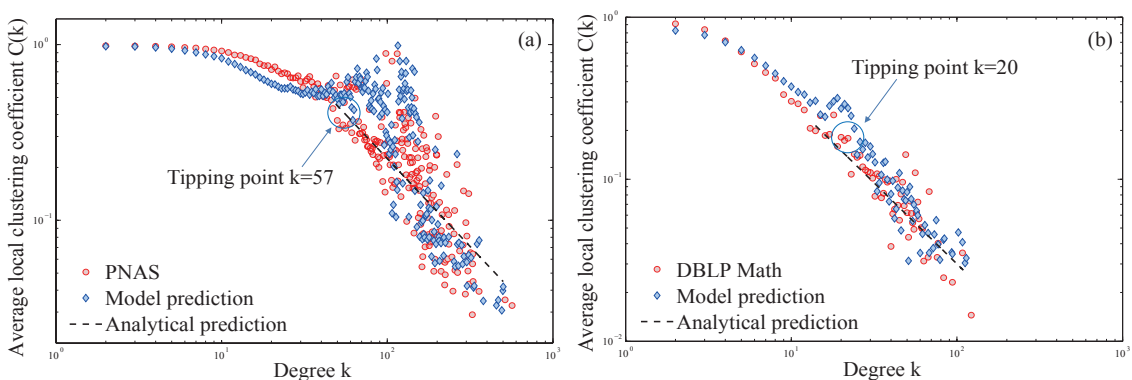

Fig. 6. The relation between local clustering coefficient and degree. The panels show the average local clustering coefficient of $k$-degree nodes for four networks in Table 2 respectively. The RMSE for the theoretical prediction $C(k) \propto 1 / k$ is 0.04597 for PNAS and 0.01476 for DBLP-Math.
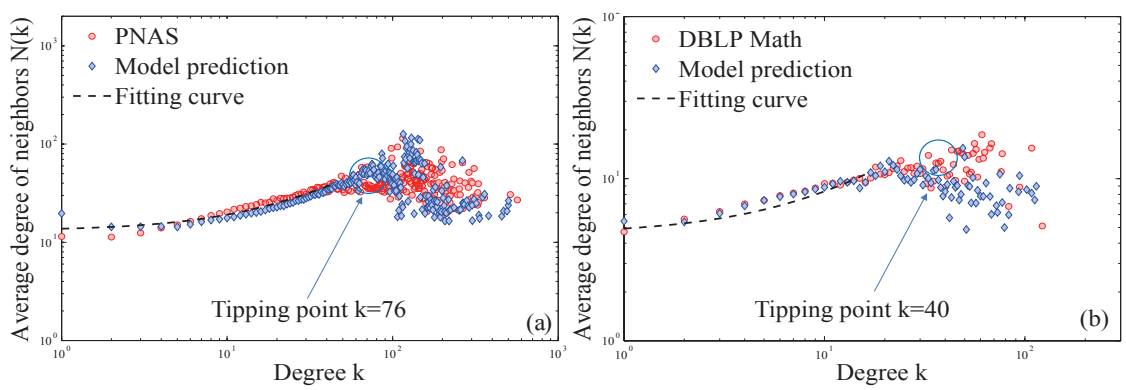

Fig. 7. The relation between degree and average degree of neighbors. The panels show $k$-degree nodes' average degree of their neighbors for four networks in Table 2 respectively. The RMSE for the linearly increasing trend is 3.075 for PNAS and 1.313 for DBLP-Math.

together, and have small degrees on average. Hence the authors in a small research team may have high local clustering coefficients and similar degrees.

As the cumulative size of a research team increases over time, the degree difference emerges between the leader and other members, because the leader usually collaborates with all members, but non-leaders only write a few papers with a few members on average. So the degrees of non-leaders, on average, do not increase with the growth of their leaders' degree, which leads to the nonpositive correlation coefficients in large $k$ regions. Meanwhile, the neighbors of non-leaders probably are non-leaders and in the same paper team. So nonleaders have high local clustering coefficients, and collaborated non-leaders have similar degrees on average, which is a reason for positive correlation coefficients in small $k$ regions. In the above analysis, the sizes of paper teams are approximated by their expected value. However, the existence of large paper teams can increase the correlation coefficients. For example, the correlation coefficients in the large $k$-regions are -0.025 and -0.045 for PNAS and 

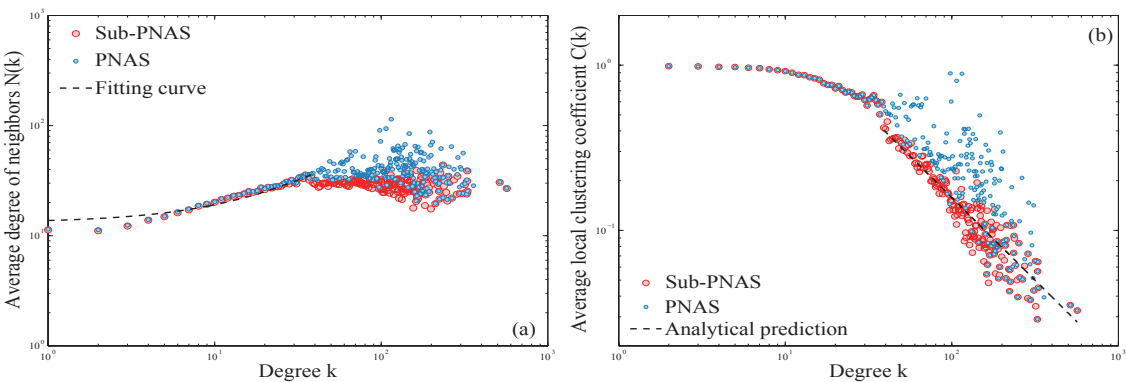

Fig. 8. The influence of large paper teams on local clustering coefficients and average degree of node neighbors. The panels show the average local clustering coefficients and average degree of neighbors for nodes with degree $k$ in Sub-PNAS and PNAS respectively.

Sub-PNAS respectively (Fig. 8 ). In addition, some non-leaders could leave their teams, and so are unlikely to collaborate with new coming members. For example, many students leave their research teams after graduation, so the students studied in different periods of time are unlikely to collaborate. The leaving also leads to the low local clustering coefficient of leaders.

Modelling the transitions

The phenomenon of research-team members leaving and that of members' activity decreasing are modeled by shrinking the influential zones. The model design leads that only those non-lead nodes very close to lead nodes in space distances could receive new "collaborators" persistently. The good model-data fit confirms the reasonability of the model design.

The analysis in Appendix shows the tails of $C(k)$ of modeled networks are proportional to $1 / k$, which are similar to those of the empirical networks (Fig. 6). This phenomenon is clear in DBLP-Math, but not quite clear in PNAS. The reason is that PNAS has few large paper teams, but the analysis in Appendix is based on the mean size of paper teams and ignores large paper teams (which occur at low-rates). The explanation can be confirmed through the tail of $C(k)$ of Sub-PNAS, which is clearly proportional to $1 / k$ (Fig. $8 \mathrm{p}$ ).

The model overcomes two fitting defects of our previous model (Xie, Ouyang: \& Li 2016), namely the functions $N(k)$ and $C(k)$ of modeled networks are more similar to those of the empirical data. For example, the increasing part of $N(k)$ here is longer than that of our previous result with the same parameter $\mu$ (see Fig. 5 in Reference (Xie, Ouyang \& Li 2016), Fig. 6), a reason of which is described as follows. Suppose node $i$ has an influence zone covers node $j$. The expected degrees of nodes $i$ and $j$ satisfy $k_{i} \approx \alpha\left(\theta_{i}\right) \delta t_{i}^{-\beta} T^{\beta} / \beta$ and $k_{j} \leq \mu \log \left(T / \max \left(m\left(\theta_{i}, t_{i}\right), t_{j}\right)\right)+\mu$ respectively. Hence the expected degree of $i$ 's neighbors is larger than that of our previous model. This also makes the degree associativity of modeled networks do not require a large $\mu$ as the 
previous model does. A small $\mu$ makes the $P(k)$ (with a small hook head) and $C(k)$ (with a smooth transition) of Modeled network 2 are all similar to those of DBLP-Math (Fig. 4b, Fig. 6b), and better than those of our previous model (see Fig. 4 in Reference (Xie, Ouyang \& Li 2016), Fig. 7).

\section{Conclusion and discussion}

The major portion of our model aims at unveiling the transition phenomena emerging in statistical properties of coauthorship networks and the mechanisms by which they are generated. It explains the emergence of transition through the different collaboration behaviors of leaders and the other teammembers of research teams. The model applies a geometric way to understand specific aspects of collaboration behaviors and provides remarkable predictions of a range of topological and statistical features of the empirical data. The model has the potential to illuminate specific views and implications in the broader study of scientific behaviors as follows.

Collaboration behaviors are essentially the self-organization decisions made by authors. In the model, those decisions made are based on the homophily (in the sense of research interests, topics, etc.) and academic impacts (with Matthew effect) of authors. The model reveals how the decisions of heterogeneous individuals in a social network generate a range of complex properties, such as scale-free and small-world. The model addresses a basic question in complexity: do there also exist inherent rules behind the social complexity? It provides an example of how pass through the divide between simplicity and complexity. The general idea of the model can be extended to explore the evolution of other complex networks generated based on human decisions, e. g. citation networks. In fact, the distribution of citations received by papers or authors, and that of papers published by authors all emerge a generated Poisson head and a power-law tail, which belong to the same distribution type of collaborators per author. Further, the view of the model potentially bridges cooperative game theory and social affiliation networks.

Which helps the development of sciences, monopoly or diversity? A strong Matthew effect drives monopoly, which will suppress diversity, and consequently harms system flexibility. Meanwhile, diversity is not to say that egalitarian resource distribution, which may be unsuitable to solve long and difficult tasks. Keeping the balance of the academic environment has the potential to guide investment directions of funding agencies and policy makers. Specific regulations can be simulated through the model to find out what will happen.

Is there any relation between the transition phenomena in citation networks (Peterson, Pressé \& Dill 2010) and those in coauthorship networks? Can we predict scientific success through collaboration behaviors? With citation information, the model can contribute to analyze the correlation and coevolution of citations and collaborations, and then can delve the extent to which authors' activity in academic society influences their academic recognition. 
The model shows the generating process of giant components in coauthorship networks and high clustering property. Now the model is restricted to two dimensional spacetime. While it is most intuitive and easiest to program, natural variations of the model will perhaps make sense in high dimensional space, e. g. subject specialty space. Then the similarity of nodes in the sense of geometric distance could simulate subject specialty. With this model, the invisible college (a set of interacting researchers who share similar research interests, even though geographically affiliated to distant research institutes (Zuccala 2016) ) could be studied by focusing on the subject specialty and researcher behaviors.

\section{References}

Adams J (2012) Collaborations: The rise of research networks. Nature 490, 335-336.

Barabási AL, Jeong H, Néda Z, Ravasz E, Schubert A, Vicsek T (2002) Evolution of the social network of scientific collaborations. Physica A 311: 590-614.

Bertsimas D, Brynjolfsson E, Reichman S, Silberholz JM (2014) Moneyball for academics: Network analysis for predicting research impact. Available at SSRN 2374581.

Blondel VD, Guillaume JL, Lambiotte R, Lefebvre E (2008) Fast unfolding of communities in large networks. J Stat Mech 10: P10008.

Börner K, et al. (2010) A multi-level systems perspective for the science of team science. Sci Transl Med 2(49): 49cm24

Börner K, Maru JT, Goldstone RL (2004) The simultaneous evolution of author and paper networks. Proc Natl Acad Sci USA 101(suppl 1), 5266-5273.

Catanzaro M, Caldarelli G, Pietronero L (2004) Assortative model for social networks. Phys Rev E 70: 037101.

Glänzel W (2011) National characteristics in international scientific co-authorship relations. Scientometrics 51: 69-115.

Glänzel W (2014) Analysis of co-authorship patterns at the individual level. Transinformacao 26: $229-238$

Glänzel W, Schubert A (2004) Analysing scientific networks through co-authorship. Handbook of quantitative science and technology research, 257-276.

Consul PC, Jain GC (1973) A generalization of the Poisson distribution. Technometrics 15(4): 791-799.

Hoekman J, Frenken K, Tijssen RJW (2010) Research collaboration at a distance: Changing spatial patterns of scientific collaboration within Europe. Res Policy 39: 662-673.

Jones BF, Wuchty S, Uzzi B (2008) Multi-university research teams: Shifting impact, geography, and stratification in science. Science 322(5905):1259-1262.

Kim J, Diesner J (2016) Distortive effects of initial-based name disambiguation on measurements of large-scale coauthorship networks. J Am Soc Inf Sci Technol 67(6):1446-1461.

Krioukov D, Kitsak M, Sinkovits RS, Rideout D, Meyer D, Boguñá M (2012) Network cosmology. Sci Rep 2: 793.

Mali F, Kronegger L, Doreian P, Ferligoj A, Dynamic scientific coauthorship networks (2012) In: Scharnhorst A, Börner K, Besselaar PVD editors. Models of science dynamics. Springer. pp. 195-232.

Milojević S (2010) Modes of collaboration in modern science-beyond power laws and preferential attachment. J Am Soc Inf Sci Technol 61(7): 1410-1423.

Milojević S (2014) Principles of scientific research team formation and evolution. Proc Natl Acad Sci USA 111: 3984-3989.

Moody J (2004) The strucutre of a social science collaboration network: disciplinery cohesion form 1963 to 1999. Am Sociol Rev 69(2): 213-238.

Newman M (2001) The structure of scientific collaboration networks. Proc Natl Acad Sci USA 98: 404-409. 
Newman M (2001) Scientific collaboration networks. I. network construction and fundamental results. Phys Rev E 64: 016131.

Newman M (2001) Scientific collaboration networks. II. shortest paths, weighted networks, and centrality. Phys Rev E 64: 016132.

Newman M (2002) Assortative mixing in networks. Phys Rev Lett 89: 208701.

Newman M (2004) Coauthorship networks and patterns of scientific collaboration. Proc Natl Acad Sci USA 101: 5200-5205.

Pastor-Satorras R, Vázquez A, Vespignani A (2001) Dynamical and correlation properties of the Internet. Phys Rev Lett 87(25): 258701.

Penrose M, Random geometric graphs, Oxford studies in probability, 2003.

Perc C (2010) Growth and structure of Slovenia's scientific collaboration network. J Informetr 4: 475-482.

Peterson GJ, Pressé S, Dill KA (2010) Nonuniversal power law scaling in the probability distribution of scientific citations. Proc Natl Acad Sci USA 107: 16023-16027.

Sarigöl E, Pfitzner R, Scholtes I, Garas A, Schweitzer F (2014) Predicting scientific success based on coauthorship networks. EPJ Data Science 3(1): 1-16.

Shrum W, Genuth J, Chompalov I (2007) Structures of Scientific Collaboration (MIT, Cambridge, MA)

Tomassini M, Luthi L (2007) Empirical analysis of the evolution of a scientific collaboration network. Physica A 285: 750-764.

Uzzi B, Mukherjee S, Stringer M, Jones B (2013) Atypical combinations and scientific impact. Science 342(6157): 468-472.

Wagner CS, Leydesdorff L (2005) Network structure, self-organization, and the growth of international collaboration in science. Res Policy 34(10): 1608-1618.

Wuchty S, Jones BF, Uzzi B (2007) The increasing dominance of teams in production of knowledge. Science 316(5827): 1036-1039.

Xie Z, Duan XJ, Ouyang ZZ, Zhang PY (2015) Quantitative analysis of the interdisciplinarity of applied mathematics. Plos One 10(9): e0137424.

Xie Z, Ouyang ZZ, Li JP (2016) A geometric graph model for coauthorship networks. J Informetr 10: 299-311.

Xie Z, Ouyang ZZ, Liu Q, Li JP (2016) A geometric graph model for citation networks of exponentially growing scientific papers. Physica A 456: 167-175.

Xie Z, Ouyang ZZ, Zhang PY, Yi DY, Kong DX (2015) Modeling the citation network by network cosmology. Plos One 10(3): e0120687.

Xie Z, Rogers T (2016) Scale-invariant geometric random graphs. Phys Rev E 93: 032310.

Zuccala A (2006) Modeling the invisible college. J Am Soc Inf Sci Technol 57(2): 152-168.

\section{Appendix}

The underlying formulae of degree distributions

Firstly, we analyze the degree distribution of modeled networks, the edges of which are only generated by Rule (a) in Step 2. The overlapping probability of zones is small, because $\alpha(\cdot)$ is small (due to the limitation of the maximum degree). Hence the overlapping of zones is ignored in the following analysis. We choose a proper $q$ to make the sizes of most hyperedges be drawn from the Poisson part $f_{1}$ with mean $\mu+1$. We initially consider the effect of those hyperedges on the degree distribution, and next consider that of the hyperedges, the sizes of which are drawn from the power-law part $f_{2}$.

Case 1: the degrees of the nodes having zones. Suppose node $i$ has a zone. Let $S(\theta)$ be the smallest $s$ satisfying $n(\theta, s, T)<\mu$. The expected degree of node $i$ contributed by Rule (a) in Step 2 is $k_{a}\left(\theta_{i}, t_{i}\right) \approx n\left(\theta_{i}, t_{i}, T\right)$ and the 
approximation holds for $t_{i} \ll T$. If $S(\theta)$ is large enough (which can be achieved by choosing proper parameters) we have a small $\left|\partial k_{a}\left(\theta_{i}, s\right) / \partial s\right|$ for $s>S(\theta)$, and so we take $k_{a}\left(\theta_{i}, s\right)$ to be independent of $s$ and write $k_{a}\left(\theta_{i}\right)$ instead of $k_{a}\left(\theta_{i}, t_{i}\right)$.

Case 2: the degrees of the nodes having no zone. Assume node $i$ is covered by a zone of node $j$. If $S\left(\theta_{j}\right) \leq t_{j} \leq T$, the expected degree of node $i$ contributed by Rule (a) in Step 2, namely by being covered by the zone of $j$, is $k_{a}\left(t_{i}, \theta_{j}, t_{j}\right) \approx n\left(\theta_{j}, t_{j}, T\right) \approx k_{a}\left(\theta_{j}\right) \approx k_{a}\left(\theta_{i}\right)$, where the third approximation is due to the small distance $d\left(\theta_{i}, \theta_{j}\right)$ and the piecewise constant property of $\alpha(\cdot)$. Now we suppose $t_{j}<S\left(\theta_{j}\right)$. Let $m\left(\theta_{j}, t_{j}\right)$ be the smallest $s$ satisfying $n\left(\theta_{j}, t_{j}, s\right)>\mu$ and $k_{a}\left(t_{i}, \theta_{j}, t_{j}, s\right)$ be the expected degree of node $i$ at time $s$. Since the nodes fall randomly and uniformly, the probability of any existing node in the current influential zone of a lead node connecting to a new node is equal. Hence the rate at which node $i$ acquires edges from the nodes coming at time $s$ satisfies

$$
\begin{aligned}
\partial k_{a}\left(t_{i}, \theta_{j}, t_{j}, s\right) / \partial s & \leq(\mu-1) \times \alpha\left(\theta_{j}\right) \delta t_{j}^{-\beta} s^{\beta-1} \times n\left(\theta_{j}, t_{j}, s-1\right)^{-1} \\
& \approx \beta(\mu-1) / s .
\end{aligned}
$$

Therefore $k_{a}\left(t_{i}, \theta_{j}, t_{j}\right) \leq \beta(\mu-1) \log \left(T / \max \left(m\left(\theta_{j}, t_{j}\right), t_{i}\right)\right)+\mu$. If $t_{i}$ is large enough, $k_{a}\left(t_{i}, \theta_{j}, t_{j}\right) \approx \mu$. In addition, $k_{a}\left(t_{i}, \theta_{j}, t_{j}\right)<\beta(\mu-1) \log (T)+\mu$ so cannot effect the tail of the degree distribution.

The degrees of nodes will not be exactly equal to their expected values because the nodes are distributed according to a Poisson point process, and so need to be averaged with the Poisson distribution. In addition, the nodes of the hyperedges with large sizes drawn from $f_{2}$ would not have small degrees. Hence the degree distribution of small degree nodes is

$$
\begin{aligned}
P_{S}(k)= & \frac{1}{2 \pi} \int_{0}^{2 \pi}\left(\frac{\epsilon(\theta) k_{a}(\theta)^{k} \mathrm{e}^{-k_{a}(\theta)}}{k !}+\frac{1-\epsilon(\theta)}{S(\theta)-1} \sum_{t=1}^{S(\theta)-1}\left(\frac{1}{T-m(\theta, t)+1}\right.\right. \\
& \left.\left.\times \sum_{s=m(\theta, t)}^{T} \frac{k_{a}(s, \theta, t)^{k} \mathrm{e}^{-k_{a}(s, \theta, t)}}{k !}\right)\right) d \theta,
\end{aligned}
$$

where $\epsilon(\theta)$ is the proportion of the nodes covered by the zones of the nodes born on or after time $S(\theta)$.

Eq. 3 is a mixture of Poisson distributions with different expected values. A generalized Poisson distribution can be well fitted by a mixture Poisson distribution with proper parameters. In fact, the probability of adding a new neighbor for a given node is affected by the space locations of previous lead nodes. Therefore, it is reasonable to consider that the predominant modeled collaborations are governed by certain generalization of Poisson processes.

The calculation for the degree distribution in large $k$ region is the same as that in Reference (Xie, Ouyang \& Li 2016), and so is briefly listed as follows:

$$
P_{L}(k)=\frac{1}{2 \pi k !} \int_{0}^{2 \pi}\left(\frac{1}{S(\theta)} \int_{1}^{S(\theta)+1} k_{a}(\theta, t, T)^{k} \mathrm{e}^{-k_{a}(\theta, t, T)} d t\right) d \theta \propto \frac{1}{k^{1+\frac{1}{\beta}}},
$$


where $k \gg 0$ is needed in the calculation.

The hyperedges with large sizes drawn from $f_{2}$ can affect the tail of degree distribution. Ignoring the overlapping of those hyperedges (which is due to the small probability of their occurrences) and the proportion of the nodes having zones and belonging to those hyperedges (which is small when compared with that of the nodes having no zone) we obtain that the degree distribution's tail of the network generated by Rule (a) in Step 2 is approximately a mixture power-law distribution $q P_{L}(k)+(1-q)(k+1) f_{2}(k+1) / \sum_{s} s f_{2}(s)$.

Finally, we analyze the degrees contributed by Rule (b). Let $k_{b}\left(\theta_{i}, t_{i}, s\right)$ be the degree of node $i$ contributed by this rule at time $s \geq t_{i}$. The number of nodes with nonzero degree at time $s$ is $N(s)=\zeta \int_{0}^{2 \pi}\left(\sum_{t=1}^{s} \alpha(\theta) \delta t^{-\beta} s^{\beta} / \beta\right) d \theta \approx$ $s \delta \zeta \int_{0}^{2 \pi} \alpha(\theta) d \theta /(\beta(1-\beta))$, where $\zeta=N_{2} /(2 \pi)$. So the probability that a node is chosen by Rule (b) at time $s$ is $(\nu+1) N_{3} / N(s)$, where $\nu+1$ is the expected value of $f$ in Rule (b). Hence, the rate at which node $i$ at time $s$ acquires edges generated by Rule (b) is $\partial k_{b}\left(t_{i}, s\right) / \partial s=\nu(\nu+1) N_{3} / N(s)$, which gives $k_{b}\left(t_{i}, T\right) \approx \beta(1-\beta) \nu(\nu+1) \log \left(T / t_{i}\right) N_{3} /\left(\delta \zeta \int_{0}^{2 \pi} \alpha(\theta) d \theta\right)$. Hence, choosing proper parameters, the degrees contributed by Rule (b) can be ignored, when compared with that contributed by Rule (a).

In simulations, the condition $k \gg 0$ required in Eq. 4 cannot be fully satisfied due to the restriction of the maximum degree, which is a reason for the difference between the theoretical value and the practical value of the power exponent. In practice, the degree distributions of the modeled networks fit the above analysis at certain levels, and are similar to those of the empirical networks (Fig. 4).

The formulae under the correlation of local clustering coefficients and degrees

Suppose node $i$ has a zone and $t_{i}$ is small enough. So the number of neighbors of node $i$ generated by Rule (b) can be ignored when compared with that generated by Rule (a). Hence the expected degree $k$ of $i$ is approximately equal to $k_{a}\left(\theta_{i}, t_{i}, T\right)$. Suppose nodes $j, l$ belong to the zone and $t_{j}<t_{l}$. Since $t_{i}$ is small, $k$ is large, and so $T-m\left(\theta_{i}, t_{i}\right) \approx T-T(\mu / k)^{1 / \beta} \approx T$, where $m\left(\theta_{i}, t_{i}\right)$ is the smallest $s$ satisfying $n\left(\theta_{i}, t_{i}, s\right)>\mu$. So we can only consider the case $t_{j}>m\left(\theta_{i}, t_{i}\right)$. Since the nodes are dropped randomly and uniformly, the probability of an edge between $j$ and $l$ is the reciprocal of the number of nodes (born before $t_{l}$ ) of the zone multiplied by the expected hyperedge size less than two, namely $\omega T^{\beta} /\left(k\left(t_{l}-1\right)^{\beta}\right)$, where the boundary effects of zones are ignored. Averaging over possible values of $t_{j}$ and $t_{l}$, the local clustering coefficient of node $i$ is

$$
\begin{aligned}
C(k) & =\frac{1}{T-m\left(\theta_{i}, t_{i}\right)} \int_{m\left(\theta_{i}, t_{i}\right)}^{T}\left(\frac{1}{T-t_{j}-1} \int_{t_{j}+1}^{T} \frac{\omega T^{\beta}}{k\left(t_{l}-1\right)^{\beta}} d t_{l}\right) d t_{j} \\
& \approx \frac{\omega T^{\beta-1}}{k(1-\beta)}\left(\int_{m\left(\theta_{i}, t_{i}\right)}^{T} \frac{(T-1)^{1-\beta}-s^{1-\beta}}{T-s-1} d s\right)
\end{aligned}
$$


where the approximation holds for $T \gg m\left(\theta_{i}, t_{i}\right)$. Denote the coefficient of $1 / k$ by $I(k)$, substitute $m\left(\theta_{i}, t_{i}\right) \approx T(\mu / k)^{1 / \beta}$ into it, and differentiate it to obtain

$$
\frac{d I(k)}{d k}=\frac{\omega T^{\beta-1}}{1-\beta} \times \frac{(T-1)^{1-\beta}-T^{1-\beta}\left(\frac{\mu}{k}\right)^{\frac{1-\beta}{\beta}}}{T-T\left(\frac{\mu}{k}\right)^{\frac{1}{\beta}}-1} \times \frac{T}{\beta} \frac{\mu^{\frac{1}{\beta}}}{k^{\frac{1}{\beta}+1}} \approx \frac{\omega \mu^{\frac{1}{\beta}}}{\beta(1-\beta) k^{\frac{1}{\beta}+1}},
$$

which is approximately equal to 0 if $k \gg \mu$. Hence $I(k)$ is free of $k$ and $C(k) \propto 1 / k$ if $k$ is large enough. The modeled networks roughly follow the above analyses (Blue diamonds in Fig. 6), in which the outliers are partly caused by the boundary effects of zones that cannot be ignored under the occurrence of some large size hyperedges drawn from $f_{2}(x)$. 